\title{
THE FIXED POINT INDEX AND ASYMPTOTIC FIXED POINT THEOREMS FOR $k$-SET-CONTRACTIONS
}

\author{
BY ROGER D. NUSSBAUM
}

\author{
Communicated by J. N. Herstein, September 27, 1968
}

1. Introduction. In $1955 \mathrm{G}$. Darbo [6] defined the measure of noncompactness, $\gamma(A)$, of a bounded subset $A$ of a metric space $(X, d): \gamma(A)=\inf \{d>0 \mid A$ can be covered by a finite number of sets of diameter less than or equal to $d\}$. If $(X, d)$ is a complete metric space, Darbo shows that for any decreasing sequence of closed, nonempty sets $A_{n}$ with $\gamma\left(A_{n}\right)$ approaching $0, \cap_{n \geq 1} A_{n}$ is compact and nonempty. If $X$ is a Banach space, Darbo also demonstrates the crucial properties $\gamma(A+B) \leqq \gamma(A)+\gamma(B)$ and $\gamma($ convex closure $A$ ) $=\gamma(A)$.

If $G$ is a subset of the metric space $X_{1}$ and $f$ is a continuous map from $G$ to a metric space $X_{2}$, Darbo calls $f$ a $k$-set-contraction if $\gamma_{2}(f(A)) \leqq k \gamma_{1}(A)$ for $A$ bounded and $A \subset G$. It is easy to show that $k$-set-contractions with $k<1$ are closed under composition and convex sums. Darbo proves that if $G$ is a closed, bounded convex subset of a Banach space $X$ and $f: G \rightarrow G$ is a $k$-set-contraction, $k<1$, then $f$ has a fixed point.

An important example of a $k$-set-contraction, $k<1$, is a map of the form $U+C, U$ a strict contraction (i.e. $\|U x-U y\| \leqq k\|x-y\|, k<1$ ) and $C$ a compact map, both defined on a subset $G$ of a Banach space $X . F$. E. Browder and the author [5] have recently defined (as a special case) a degree theory for mappings of the form $I-U-C$, so it is natural to ask if one can obtain a degree theory for mappings of the form $I-f, f$ a $k$-set-contraction, $k<1$. In fact we will define a fixed point index for $k$-set-contractions on certain nice ANR's, and we will give direct generalizations of all properties of the classical fixed point index.

In another direction let $X$ be a bounded, complete metric space and $f: X \rightarrow X$ a $k$-set-contraction, $k<1$. Using Darbo's results we can prove that $\operatorname{cl}\left(\bigcap_{n \geq 1} f^{n}(X)\right)$ is nonempty and compact. In general Browder [3] has suggestively called such maps asymptotically compact and has proved fixed point theorems about them. Such theorems have proved useful in studying ordinary differential equations. We generalize Browder's chief result to the context of $k$-set-contractions.

2. The fixed point index for $k$-set-contractions. Let us begin by recalling the basic properties of the classical fixed point index. Let $X$ 
be a compact, metric absolute neighborhood retract (ANR) and $G$ an open subset of $X$. Let $f: G \rightarrow X$ be a continuous function and assume the fixed point set of $f$ in $G$ is compact (it may be empty). Then we can define an integer $i_{X}(f, G)$ which has the following properties (all spaces here are compact, metric ANR's):

(a) Let $I$ denote the closed unit interval $[0,1]$ and let $\Omega$ be an open subset of $X \times I$. Let $F: \Omega \rightarrow X$ be a continuous map and assume that $\{(x, t) \mid F(x, t)=x\}$ is a compact subset of $\Omega$. Let $\Omega_{t}=\{x \mid(x, t) \in \Omega\}$ and $F_{t}=F(\cdot, t)$. Then we have $i_{X}\left(F_{0}, \Omega_{0}\right)=i_{X}\left(F_{1}, \Omega_{1}\right)$ (the homotopy property).

(b) Let $f: G \rightarrow X$ be a continuous function and assume that $S$ $=\{x \mid f(x)=x\}$ is a compact subset of $G$. Let $G_{1}$ and $G_{2}$ be disjoint open subsets of $G$ such that $S \subset G_{1} \cup G_{2}$. Then we obtain $i_{X}(f, G)$ $=i_{X}\left(f, G_{1}\right)+i_{X}\left(f, G_{2}\right)$ (the additivity property).

(c) Let $f: X \rightarrow X$ be a continuous function and let $\Lambda(f)$ denote the Lefschetz number of $f$. Then we have $i_{X}(f, X)=\Lambda(f)$ (the normalization property).

(d) Let $X_{1}$ and $X_{2}$ be compact, metric ANR's, $G_{1}$ and $G_{2}$ open subsets of $X_{1}$ and $X_{2}$ respectively. Suppose $f_{1}: G_{1} \rightarrow X_{2}$ and $f_{2}: G_{2} \rightarrow X_{1}$, so that $f_{2} f_{1}: f_{1}^{-1}\left(G_{2}\right) \rightarrow X_{1}$ and $f_{1} f_{2}: f_{2}^{-1}\left(G_{1}\right) \rightarrow X_{2}$. Assume that

$$
S_{1}=\left\{x \in \overline{f_{1}}\left(G_{2}\right) \mid\left(f_{2} f_{1}\right)(x)=x\right\}
$$

is compact. Then $S_{2}=\left\{x \in f_{2}^{-1}\left(G_{1}\right) \mid\left(f_{1} f_{2}\right)(x)=x\right\}$ is compact and $i_{X_{1}}\left(f_{2} f_{1}, f_{1}^{-1}\left(G_{2}\right)\right)=i_{X_{2}}\left(f_{1} f_{2}, f_{2}^{-1}\left(G_{1}\right)\right)$ (the commutativity property).

The four properties listed here are slight variants of the usual properties proved in the literature for the fixed point index. They can be proved without too much difficulty.

Let us introduce some notation. Let $X$ be a closed subset of a Banach space $B$. We shall say $X \in \mathcal{F}$ if we can write $X=\bigcup_{i=1}^{n} C_{i}$, where $C_{i}$ are closed, convex sets in $B$. The metric on $X$ will always be that which it inherits from $B$. Actually, the following results hold if we only know that $X$ is a locally finite union of closed, convex sets, i.e. $X=\mathrm{U}_{\alpha \in \Lambda} C_{\alpha}$ and every $x \in X$ has a neighborhood $N_{x}$ such that $N_{x} \cap C_{\alpha}=\varnothing$ except for finitely many $\alpha$.

Let $G$ be a subset of a Banach space $B$ and $f: G \rightarrow B$ a continuous map. Let us write $K_{1}(f, G)=\operatorname{cocl} f(G), K_{n}(f, G)=\operatorname{cocl} f\left(G \cap K_{n-1}(f, G)\right.$ ), and $K_{\infty}(f, G)=\bigcap_{n \geq 1} K_{n}(f, G)$; cocl denotes convex closure. It is easy to see that $f: G \cap K_{\infty}(f, G) \rightarrow K_{\infty}(f, G)$ and $K_{\infty}(f, G)$ is closed and convex. If $G$ is bounded and $f: G \rightarrow X$ is a $k$-set-contraction, $k<1$, Darbo's results also imply that $K_{\infty}(f, G)$ is compact.

Suppose that $X \in \mathcal{F}, G$ is an open subset of $X$ and $f: G \rightarrow X$ is a continuous map. Assume that $S=\{x \in G \mid f(x)=x\}$ is compact. Fi- 
nally, assume that $f$ is a local strict-set-contraction. By this we mean that every point $x \in G$ has a neighborhood $N_{x}$ such that for $D \subset N_{x}$, $\gamma(f(D)) \leqq k_{x} \gamma(D), k_{x}<1$. Using these assumptions, we can find a bounded open neighborhood $G_{1}$ of $S$ such that $f: G_{1} \rightarrow X$ is a $k$-setcontraction, $k<1$. Let us write $K_{\infty}^{*}=K_{\infty}\left(f, G_{1}\right) \cap X ; K_{\infty}^{*}$ is a compact, metric ANR, $G_{1} \cap K_{\infty}^{*}$ is an open subset of $K_{\infty}^{*}$, and $f: G_{1} \cap K_{\infty}^{*} \rightarrow K_{\infty}^{*}$ is a continuous function satisfying the necessary condition, so $i_{K_{\infty}^{*}}\left(f, G_{1} \cap K_{\infty}^{*}\right)$ is defined. We define $i_{X}(f, G)=i_{K_{\infty}^{*}}\left(f, G_{1} \cap K_{\infty}^{*}\right)$. All the usual properties carry through to this setting.

(a) $i_{X}(f, G)$ does not depend on the particular $G_{1}$ chosen. Further, if $X$ is a compact metric ANR, $i_{X}(f, G)$ (the usual definition) equals $i_{K_{\infty}^{*}}\left(f, G_{1} \cap K_{\infty}^{*}\right)$.

(b) Let $I=[0,1]$ and let $\Omega$ be an open subset of $X \times I, X \in$ F. Let $F: \Omega \rightarrow X$ be a continuous map and assume that $\{(x, t) \mid F(x, t)=x\}$ is compact. Assume that $F$ is a local strict-set-contraction in the following sense: Given $\left(x_{0}, t_{0}\right) \in \Omega$, we can find an open neighborhood $N_{\left(x_{0}, t_{0}\right)} \subset \Omega$ of $\left(x_{0}, t_{0}\right)$ such that for $D \subset X, \gamma\left(F\left(N_{\left(x_{0}, t_{0}\right)} \cap(D \times I)\right)\right)$ $\leqq k_{\left(x_{0}, t_{0}\right)} \gamma(D), k_{\left(x_{0}, t_{0}\right)}<1$. Then $i_{X}\left(F_{t}, \Omega_{t}\right)$ is defined for $t \in I$ and $i_{X}\left(F_{0}, \Omega_{0}\right)=i_{X}\left(F_{1}, \Omega_{1}\right)$ (the homotopy property).

If $\Omega$ is of the form $G \times I$, where $G$ is a bounded, open set, and $F$ is defined on $\operatorname{cl}(G) \times I$, then if $F(x, t) \neq x$ for $x \in \partial G$ and $\gamma(F(A \times I))$ $\leqq k \gamma(A), k<1$, for $A \subset \operatorname{cl}(G)$, the conditions of the homotopy property are met. This latter condition is satisfied if each $F_{t}: \operatorname{cl}(G) \rightarrow X$ is a $k$-set-contraction, $k<1, k$ independent of $t$, and the map $t \rightarrow F_{t}$ is continuous from $I$ to the sup topology for bounded, continuous functions on $G$.

(c) Let $G$ be an open subset of a space $X \in \mathcal{F}$ and let $f: G \rightarrow X$ be a local strict-set-contraction. Assume $S=\{x \in G \mid f(x)=x\}$ is compact and $S \subset G_{1} \cup G_{2}$, where $G_{1}$ and $G_{2}$ are disjoint open subsets of $G$. Then we have $i_{X}(f, G)=i_{X}\left(f, G_{1}\right)+i_{X}\left(f, G_{2}\right)$ (the additivity property).

(d) Assume $X \in F$ and let $f: X \rightarrow X$ be a $k$-set-contraction, $k<1$. Suppose that $f^{n}(X)$ is bounded for some $n$. Then $i_{X}(f, X)$ and $\Lambda_{\text {gen }}(f)$ are defined and $i_{X}(f, X)=\Lambda_{\text {gen }}(f)$, where $\Lambda_{\text {gen }}(f)$ denotes the generalized Lefschetz number defined by Leray in [8] (the normalization property).

(e) Let $G_{1}$ and $G_{2}$ be open subsets of spaces $X_{1}$ and $X_{2}, X_{i} \in F$. Let $f_{1}: G_{1} \rightarrow X_{2}$ and $f_{2}: G_{2} \rightarrow X_{1}$ be, respectively, $k_{1}$ - and $k_{2}$-set-contractions, $k_{1} k_{2}<1$. If $f_{1}$ is a 0 -set-contraction, we only need to assume $f_{2}$ is continuous. Let $S_{1}=\left\{x \in f_{1}^{-1}\left(G_{2}\right) \mid\left(f_{2} f_{1}\right)(x)=x\right\}$ and assume that $S_{1}$ is compact. It follows then that $S_{2}=\left\{x \in f_{2}^{-1}\left(G_{1}\right) \mid\left(f_{1} f_{2}\right)(x)=x\right\}$ is compact and $i_{X_{1}}\left(f_{2} f_{1}, f_{1}^{-1}\left(G_{2}\right)\right)=i_{X_{2}}\left(f_{1} f_{2}, f_{2}^{-1}\left(G_{1}\right)\right)$ (the commutativity property). 
The above properties can be derived from the corresponding results for the classical fixed point index with the aid of the following lemma.

LEMMA 1. Let $A=\mathrm{U}_{i=1}^{n} C_{i}$ be a finite union of compact, convex sets $C_{i}$ in a Banach space $X$. Let $B=\bigcup_{i-1}^{n} D_{i}$ be another finite union of compact, convex sets with $C_{i} \supset D_{i}, 1 \leqq i \leqq n$. Let 0 be an open subset of $A$ and $f$ : $\operatorname{cl}(0) \rightarrow A$ a continuous map such that $f(x) \neq x$ for $x \in \mathrm{cl}(0)-0$. Assume that $f: 0 \cap B \rightarrow B$ and that $B \supset K_{\infty}(f, 0) \cap A$. Then $i_{A}(f, 0)=i_{B}(f, 0 \cap B)$.

Lemma 1 , in turn, is proved with the aid of a purely geometrical result which may have some independent interest.

Lemma 2. Let $A_{n}=\mathrm{U}_{i=1}^{m} C_{n}^{t}$, where $m$ is independent of $n, C_{n}^{t}$ is a compact, convex set in a Banach space $X$, and $C_{n}^{i} \supset C_{n+1}^{t}, 1 \leqq i \leqq m$. Let $A_{\infty}=\bigcap_{n \geq 1} A_{n}$. Then given $\delta>0, A_{\infty} \subset A_{n}$ is a deformation retract of $A_{n}$ for $n \geqq n(\delta)$ and the deformation retraction $H_{n}: A_{n} \times I \rightarrow A_{n}$ can be chosen so that $\left\|H_{n}(x, t)-x\right\|<\delta$ for $(x, t) \in A_{n} \times I$.

3. Degree theory for $k$-set-contractions in Banach space. Let $G$ be a bounded open subset of a Banach space $X, I$ the identity on $X$, and $f: \operatorname{cl}(G) \rightarrow X$ a $k$-set-contraction, $k<1$. Assume that $(I-f)(x) \neq a$ for $x \in \partial G$. Then we define $\operatorname{deg}(I-f, G, a)=i_{X}(f+a, G)$. As one might suspect, when $f=U+C, U$ a strict contraction, $C$ compact, this definition agrees with that given by Browder and Nussbaum [5].

As a trivial application of the above apparatus, we find the following simple refinement of Darbo's theorem.

ThEOREM. Let $G$ be a bounded, closed, convex set with nonempty interior. Assume $f(\partial G) \subset G$. Then $f$ has a fixed point.

Since Leray has proved an invariance of domain theorem for mappings of the form $I-C, C$ compact, one might also hope for such a theorem for maps of the form $I-f, f$ a local strict-set-contraction. This turns out to be true.

Theorem (INVARIANCE OF DOMAIN). Let $G$ be an open subset of $a$ Banach space $X$ and $f$ a local strict-set-contraction, $f: G \rightarrow X$. Assume $(I-f)$ is one to one. Then $(I-f)(G)$ is open.

The principal lemma for proving the above theorem is the following result. For the case that $f$ is a compact map, this is a classical theorem.

THEOREM. Let $B$ be a closed ball about the origin in a Banach space $X$ and $f: B \rightarrow X$ a $k$-set-contraction, $k<1$. Assume that $f(x) \neq x$ for $x \in \partial B$ and that $f(-x)=-f(x)$ for $x \in \partial B$. Then we have $\operatorname{deg}(I-f$, int $B, 0) \neq 0$.

We also obtain results in other directions. If $X$ is a uniformly con- 
vex Banach space, $G$ a closed, bounded, convex subset of $X$, and $f: G \rightarrow X$ continuous, say that $f$ satisfies condition $C C$ if for any $x \in G$ and $\epsilon>0$ we can find a weak neighborhood $N_{x}$ of $x$ in $G$ such that for $u, v \in N_{x},\|f(u)-f(v)\| \leqq\|u-v\|+\epsilon$. As an example, let $V$ be a nonexpansive map, $C$ a completely continuous map, both defined on $G$. Let $U$ be a nonexpansive map defined on $(V+C)(G)$. Then $U(V+C)$ satisfies condition $C C$. If $f$ satisfies condition $C C, f$ is a 1 -set-contraction.

Theorem. Let $f, G$, and $X$ be as above. Assume $f: G \rightarrow G$ (or $f: \partial G \rightarrow G$ if $G$ has nonempty interior). Then $f$ has a fixed point.

This is a generalization of some results of Browder [4] and Kirk [7].

4. Asymptotic fixed point theorems for $k$-set-contractions. In a recent article [3], Browder has proved a slight variant of the following theorem.

BROWDER's THEOREM. Let $X$ be a metrizable, locally completely metrizable ANR. Let $f: X \rightarrow X$ be a continuous map. We make the following assumptions about $f$ :

(a) $\cap_{n \geq 1} f^{n}(X)$ is nonempty and has compact closure in $X$.

(b) $f$ is locally compact.

(c) $\operatorname{cl}\left(\bigcap_{n \geq 1} f^{n}(X)\right)$ is homologically trivial in some compact set $K \subset X$, while for $z \in X, \bigcup_{j \geq 0} f_{i}(z)$ has compact closure. Then $f$ has a fixed point.

We prove the following result, which can be shown (with some effort) actually to include Browder's theorem.

ThEOREM. Let $G$ be an open subset of a space $X \in \mathcal{F}$. Let $f: G \rightarrow G$ be a continuous map. We make the following assumptions about $f$ :

(a) $\bigcap_{n \geq 1} f^{n}(G)$ is nonempty and has compact closure in $G$.

(b) $f$ is a local strict-set-contraction.

(c) There is a compact set $K \supset \operatorname{cl}\left(\cap_{n \geq 1} f^{n}(G)\right)$ such that $\operatorname{cl}\left(\bigcap_{n \geq 1} f^{n}(G)\right)$ is homologically trivial in $K$ and such that $U_{n \geq 0} f^{n}(K)$ has compact closure in $G$. Then we find $i_{X}(f, G)$ is defined and nonzero. In particular, $f$ has a fixed point.

The fact that $i_{X}(f, G) \neq 0$ is interesting methodologically, for it suggests that the proofs of asymptotic fixed point theorems are methods of proving a generalized fixed point index is not zero.

As corollaries of the above theorem we can obtain simpler but more elegant results.

ThEOREM. Let $X \in \mathcal{F}$ and $f: X \rightarrow X$ be a $k$-set-contraction, $k<1$. Assume that $f^{n}(X)$ is bounded for some n. It follows that $\bigcap_{n \geq 1} f^{n}(X)$ is non- 
empty and has compact closure $C_{\infty}$. Assume that $C_{\infty}$ is homologically trivial in some compact set $K \supset C_{\infty}$. Then $i_{x}(f, X) \neq 0$ and $f$ has a fixed point.

Theorem. Let $X$ be a closed, convex subset of a Banach space $B$. Let $f: X \rightarrow X$ be a $k$-set-contraction, $k<1$. Assume that $f^{n}(X)$ is bounded for some $n$. Then $i_{X}(f, X) \neq 0$, and $f$ has a fixed point.

This last theorem is a direct generalization of one of the earliest asymptotic fixed point theorems [2]: Let $X$ be a Banach space and $C: X \rightarrow X$ a continuous map which is compact on bounded sets. Assume that $C^{n}(X)$ is bounded. Then $C$ has a fixed point.

\section{REFERENCES}

1. F. E. Browder, On the fixed point index for continuous mappings of locally connected spaces, Summa Brasil. Math 4 (1960), 253-293.

2. - On a generalization of the Schauder fixed point theorem, Duke Math. J. 26 (1959), 291-303.

3. - Asymptotic fixed point theorems, Math. Ann. (to appear).

4. - Semicontractive and semiaccretive nonlinear mappings in Banach spaces, Bull. Amer. Math. Soc. 74 (1968), 660-666.

5. F. E. Browder and R. D. Nussbaum, The topological degree for noncompact, non-linear mappings in Banach spaces, Bull. Amer. Math. Soc. 74 (1968), 671-677.

6. G. Darbo, Punti uniti in trasformazioni a condominio non compatto, Rend. Sem. Mat. Univ. Padova 24 (1955), 84-92.

7. W. A. Kirk, $A$ fixed point theorem for mappings which do not increase distance, Amer. Math. Monthly 72 (1965), 1004-1006.

8. J. Leray, Theorie des points fixes, indice total et nombre de Lefschetz, Bull. Soc. Math France 87 (1959), 221-233.

9. M. Nagumo, Degree of mappings in convex linear topological spaces, Amer. J. Math 73 (1951), 497-511.

University of Chicago, Chicago, Illinois 60637 